\title{
Atomic-Scale Sliding Friction on Graphene in Water
}

\author{
J. G. Vilhena, ${ }^{\dagger, \|}$ Carlos Pimentel, ${ }^{\ddagger, \perp}$ Patricia Pedraz, ${ }^{\S}$ Feng Luo, ${ }^{\S}$ Pedro A. Serena, ${ }^{\dagger}$ Carlos M. Pina, ${ }^{\ddagger}, \perp$ \\ Enrico Gnecco, ${ }^{\S, \#}$ and Rubén Pérez ${ }^{*, \|, \square}$
}

\begin{abstract}
${ }^{\dagger}$ Instituto de Ciencia de Materiales de Madrid (ICMM), CSIC, c/Sor Juana Ines de la Cruz 3, E-28049 Madrid, Spain
${ }^{\ddagger}$ Departamento de Cristalografía y Mineralogía, Universidad Complutense de Madrid, E-28040 Madrid, Spain

${ }^{\S}$ Instituto Madrileño de Estudios Avanzados, IMDEA Nanociencia, Calle Faraday 9, E-28049 Madrid, Spain

"Departamento de Física Teórica de la Materia Condensada, Universidad Autónoma de Madrid, E-28049 Madrid, Spain

${ }^{\perp}$ Instituto de Geociencias (CSIC, UCM), E-28040 Madrid, Spain

Otto Schott Institute of Materials Research, Friedrich Schiller University Jena, D-07742 Jena, Germany

Condensed Matter Physics Center (IFIMAC), Universidad Autónoma de Madrid, E-28049 Madrid, Spain
\end{abstract}

\section{Supporting Information}

\begin{abstract}
The sliding of a sharp nanotip on graphene completely immersed in water is investigated by molecular dynamics (MD) and atomic force microscopy. MD simulations predict that the atomicscale stick-slip is almost identical to that found in ultrahigh vacuum. Furthermore, they show that water plays a purely stochastic role in sliding (solid-to-solid) friction. These observations are substantiated by friction measurements on graphene grown on $\mathrm{Cu}$ and $\mathrm{Ni}$, where, oppositely of the operation in air, lattice resolution is readily achieved. Our results promote friction force microscopy in water as a robust alternative to ultra-high-vacuum measurements.
\end{abstract}

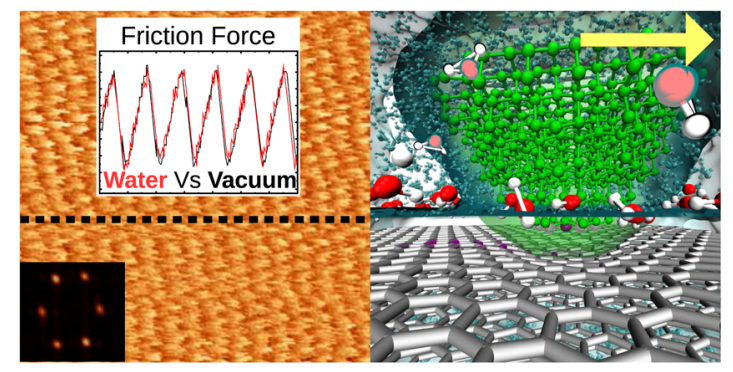

KEYWORDS: friction, nanoscale, friction in water, friction force microscopy, nanoasperity, lattice resolution, graphene, molecular dynamics, steered molecular dynamics, vacuum, water, hydration layers

Since friction is pivotal in everyday life, it is quite surprising that our understanding of the atomistic processes occurring when two surfaces slide past each

other is still quite limited. This can be attributed to the complexity of molecular-scale modeling of friction and to the lack of reproducible measurements with high spatial resolution in well-defined environmental conditions. A major breakthrough came with the rise of atomic force microscopy (AFM) in the 1980 s. $^{1}$ In this technique a sharp tip is elastically driven on a solid substrate, resembling the sliding of a single asperity over a rough surface, and the time variations of the friction force arising at the interface are recorded. The first latticeresolved friction maps of graphite ${ }^{2}$ (with normal force values in the $\mu \mathrm{N}$ range) revealed that binding and unbinding of atoms at the contacting surfaces occur in a stick-slip fashion. However, lattice resolution in ambient conditions can be made difficult by the presence of contaminants and by water bridges that are possibly formed between tip and surface and are dragged during the scanning. ${ }^{3}$ This last effect can be avoided in ultrahigh vacuum (UHV), where, using ultrasharp tips as AFM probes, subnanometer resolution of complex structures can be routinely achieved in lateral force maps. ${ }^{4-7}$
An alternative approach to avoid capillary condensation is to completely immerse the tip and the sample in water. Lattice resolution on insulating surfaces using AFM was indeed reported for the first time on calcite in water. ${ }^{8}$ In spite of this promising result, friction force microscopy (FFM) in water has been rarely explored afterward. Labuda et al. ${ }^{9}$ obtained high resolution on gold in perchloric acid, whereas some of us have recently reported lattice-resolved FFM images on calcite and dolomite (104) surfaces $^{10}$ and on ultrathin films of $\mathrm{CuPc}$ molecules self-assembled on dolomite $(104) .{ }^{11}$ Note that, in the last work, the same tip could resolve both the hydrophilic mineral substrate and the hydrophobic organic adsorbate. This leads to question the role played by water molecules in FFM and whether such high resolution is just a fortuitous event or it represents a practical alternative to UHV operation.

Graphene is an ideal material to show the potential of measuring friction in water. Many of the proposed technological applications, ${ }^{12}$ e.g., sensing devices, must take into account its mechanical response in a liquid environment. On 
the other hand, the low friction experienced on graphene makes it a challenging test for the FFM-in-liquids technique. To the best of our knowledge, the nanotribology of this material in liquid has been investigated in FFM measurements only by Robinson et al., ${ }^{13}$ who characterized a few monolayers of graphene in water and in a nonpolar liquid without achieving lattice resolution. A recent combined FFM and MD study ${ }^{14}$ concluded that the presence of a small amount of water increases friction at graphite surface steps.

Here, we perform FFM measurements on graphite, G/Ni, and $\mathrm{G} / \mathrm{Cu}$ in water and show that their surface lattices are easily resolved in water. The choice of the last two systems is motivated by two recent works by Egberts et al. ${ }^{15}$ and Paolicelli et $a .^{16}$, who measured similar surfaces using FFM in lowvacuum nitrogen and in air conditions, respectively, without achieving lattice resolution. The experimental results are supported by molecular dynamics (MD) simulations, allowing us to shed light on the intrinsic mechanisms ruling atomic-scale sliding friction in water that are not accessible by FFM. The MD simulations predict that, for the load values at which lattice resolution is achieved in the experiment, tip and substrate are in full contact, and atomic-scale stick-slip is expected to be almost identical to that found in UHV. The characterization of the sliding with long simulation times, large unit cells, and small tip velocities conclusively shows that water loses its force memory much faster than the sliding of the tip, and therefore it plays a purely stochastic role in the FFM measurements. In this way, one can transpose the results of other theoretical and experimental characterization of graphene and, in principle, of any other hydrophobic surface, as obtained in UHV, to more realistic working conditions without loss of generality. Quite interestingly, we also predict contrast reversal when the friction force is mapped on a single water monolayer dynamically ordered over graphene, although the noise level of our instrument does not allow an experimental validation of this conclusion.

\section{RESULTS AND DISCUSSION}

In Figure 1 we show the FFM measurements obtained with tip and sample fully embedded in water. Figure 1A shows an AFM lateral force map of two graphene islands partially covering the $\mathrm{Cu}$ substrate. The islands are grown across the step edges of the substrate in a carpet-like fashion. The surface lattice of graphene is clearly visible in a high-resolution force map acquired on a $5 \mathrm{~nm}$ large area at a normal load of $50 \mathrm{nN}$ (Figure 1B). From the fast Fourier transform (FFT) of the lateral force map, a lattice constant of $0.23 \mathrm{~nm}$ is estimated. The contrast was significantly reduced in the corresponding topography image (not shown), and no lattice resolution could be achieved in ambient conditions using the same probing tip. The cross-section corresponding to forward and backward scans along the dashed line in Figure 1B is displayed in Figure 1C. It shows that the contrast is caused by the stickslip motion of the tip, which moves on the substrate discontinuously. Stick-slip is still clearly visible with significantly smaller loads (6 and $11 \mathrm{nN}$; see Figure $\mathrm{S} 1$ in the Supporting Information). Measurements on graphene on $\mathrm{Ni}$ (Figure 1D) reproduce the same trend: clear lattice resolution on the force map, which is significantly reduced on the topography and completely lost in ambient conditions. On graphene on $\mathrm{Cu}$ we have also measured the average value of the lateral force $\left(F_{\mathrm{L}}\right)$ as a function of the normal force $F_{\mathrm{N}}$ at increasing and decreasing values of $F_{\mathrm{N}}$. No hysteresis is

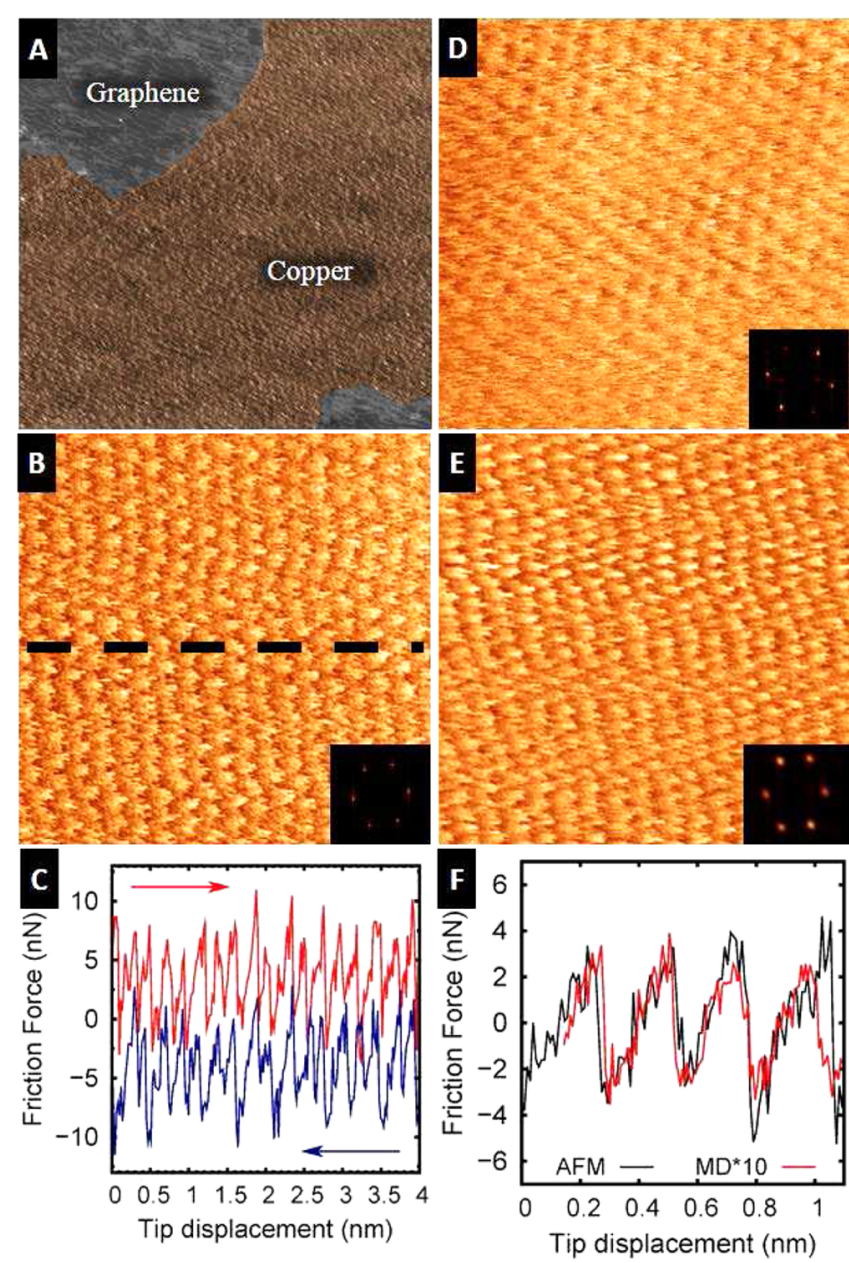

Figure 1. Friction measurements in water. (A) AFM lateral force map in water $\left(13.17 \times 13.7 \mu \mathrm{m}^{2}\right)$ of graphene islands on $\mathrm{Cu}$. Normal force $F_{\mathrm{N}}=50 \mathrm{nN}$. (B) High-resolution lateral force map (4 $\times 4 \mathrm{~nm}^{2}$ ) on an island and 2D FFT (inset). (C) Cross-section corresponding to forward and backward scans along the line in (B). (D) Same as (B) for Gr/Ni. (E) Same as (B) for graphite. (F) Comparison between friction force of $\mathrm{Gr} / \mathrm{Cu}$ in water obtained in the experiments at $F_{\mathrm{N}}=6 \mathrm{nN}$ and in our $\mathrm{MD}$ simulations at $F_{\mathrm{N}}=5$ $\mathrm{nN}$ rescaled by a factor of 10 (see text).

observed, and the friction coefficient, defined as the slope of the corresponding $F_{\mathrm{L}}-F_{\mathrm{N}}$ curves, is $\mu=0.065 \pm 0.010$. For comparison $\mu=0.36 \pm 0.02$ on $\mathrm{Cu}$ and $0.048 \pm 0.007$ on a graphite surface, which was also measured in water (Figure 1E). The low value of friction on graphene and its decrease with increasing number of layers, i.e., on graphite, are consistent with recent measurements in UHV with comparable resolution. ${ }^{17}$

FFM-MD simulations have proven to be essential in explaining a manifold of complex physical processes. ${ }^{18,19}$ The best experimental comparison with simulation is achieved, in particular, when experiments are performed under UHV conditions because MD simulations do not contain an atmosphere. $^{14}$ When MD simulations are transposed to water, a major problem stems from the large (10-fold) increase of the number of atoms due to the explicit inclusion of all water molecules.

In this work, we have performed constant-velocity steered molecular dynamics (SMD) simulations of both the sliding and the indentation of a diamond tip on supported graphene in two different environments, i.e., vacuum and water, using the 
AMBER software suite with NVIDIA GPU acceleration. ${ }^{20,21}$ As substrate, we have chosen a three-layer graphene slab, where the bottom layer is kept fixed to mimic the effect of the rigid metal supports used in our experiments. Both indentation and sliding friction forces are calculated from SMD runs using appropriate constraints (see Figure S2), and they naturally include entropic effects (see Methods and the Supporting Information for details). With the AMBER package, we not only circumvent the problem associated with simulating much larger systems but are also able to scan with velocities that are 100 times slower $(0.001 \mathrm{~m} / \mathrm{s})$ with the same computational cost of previously used methods. ${ }^{19,22-24}$ These velocities, although still much larger than the ones used in the experiments, are low enough to stay in the regime of thermally activated sliding. ${ }^{24}$ In addition, since AMBER is a biochemistry simulation suite, one can easily describe more complex biological systems such as proteins and DNA, thus providing the necessary framework for studying bio-nanotribology.

Figure 2 shows the friction curves obtained at three normal force values as simulated under vacuum and in water. In vacuum (Figure 2A) we observe that already at a small load value as $5 \mathrm{nN}$ the friction curve has the sawtooth shape characteristic of the stick-slip mechanism. Inspecting both the dynamics and the distance between force minima $(\sim 2.4 \AA)$, one realizes that the tip is pinned by the hollow sites of graphene. Furthermore, the mean friction obtained at different normal loads not only is relatively low but also barely increases with $F_{N}$, as observed in our experiments. These trends are consistent with previous experimental ${ }^{4,25}$ and theoretical ${ }^{23}$ work in UHV.

Strikingly, these same results are reproduced by the simulations in water. Not only do we observe the same stick-slip behavior obtained in vacuum, ${ }^{23,25}$ but also the contrast turns out to be similar in the two environments. This agreement is better appreciated in Figure 2C, which shows that the only remarkable difference between vacuum and water friction curves is the larger fluctuations on the latter around otherwise similar values. Furthermore, our result at the lowest load $\left(F_{\mathrm{N}}=5 \mathrm{nN}\right)$, simply multiplied by a factor of 10 , matches the periodicity and friction values measured in the experiments (Figure 1F). This rescaling factor, which takes into account the difference in contact area between the simulations and the experiments, is reasonable, given the differences in tip shape and material (a harder diamond tip in the calculations), the slightly larger operation load in the experiments, and that no attempt has been made to match the relative tip/surface orientation.

In order to understand the negligible effect of water on the friction contrast, we need to address two issues: the possible role of water in the contact region and the effect of the fluctuations induced by the fast collisions occurring between the tip and the surrounding water molecules. To answer the first question, we have to consider the contribution of hydration layers to the tip-sample interaction. Figure $3 \mathrm{~A}-\mathrm{C}$ show snapshots of the tip approaching the surface at different loads. We can observe that for a relatively small load like $F_{\mathrm{N}}=5 \mathrm{nN}$ (Figure 3C), which shows clear stick-slip in Figure 2, the tip is already in direct contact with the surface. We can understand this result comparing the normal force during the tip approach in both environments (Figure 3D). In vacuum (blue curve), three interaction regimes can be distinguished: weakly interacting $(d>6.5 \AA)$, attractive $(6.5<d<3.3 \AA)$, and repulsive $(d<3.3 \AA)$. In the water case (red curve), superimposed to these regimes, one can clearly distinguish
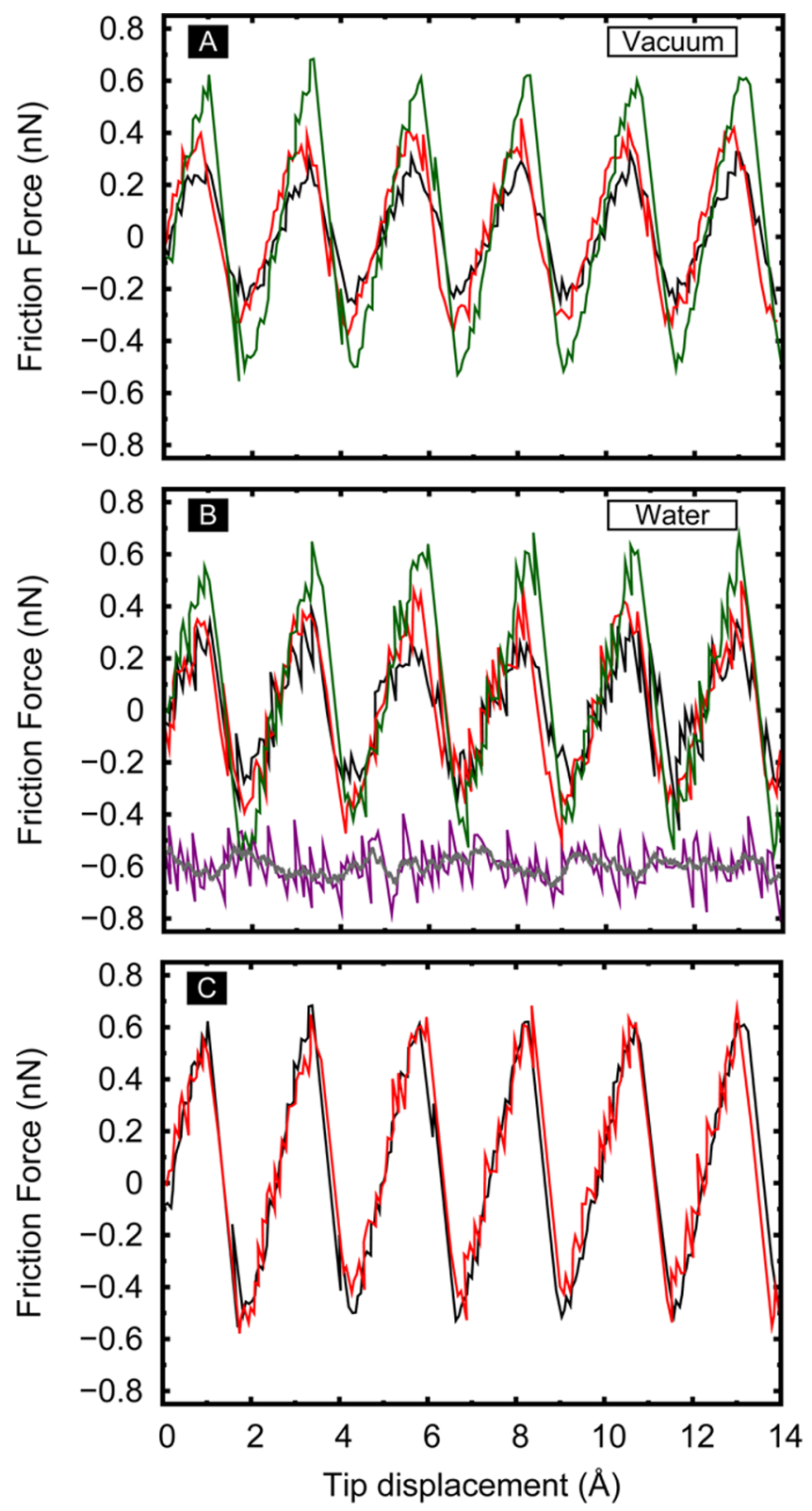

Figure 2. (A) MD simulation friction force as a function of the sliding distance under vacuum. The black, green, and red curves correspond to friction curves measured at different normal loads, i.e., 5, 10, and $20 \mathrm{nN}$, respectively. (B) Same meaning as (A), but friction is now measured with the system (tip and surface) fully embedded in water. Additionally, the purple and gray curves show a friction curve obtained by scanning over the first hydration layer and, respectively, its moving average over $0.1 \mathrm{~nm}$. For clarity reasons the last two curves have been shifted by $-0.6 \mathrm{nN}$. (C) Comparison between the friction curves obtained at a normal load of $20 \mathrm{nN}$ under vacuum (black curve) and in water (red curve).

two peaks on the force curve. These peaks arise due to the breaking of the second and first hydration layers known to form over graphene. $^{26-28}$ This process is illustrated in Figure 3A and $B$ for the first hydration layer (see Figures S3 and S4 in the Supporting Information for further details). The position of the surface hydration layers measured in our MD simulations is in agreement with specular X-ray data. ${ }^{27}$ At variance with the surface, we do not see such well-defined hydration layers around our tip. This result complies with the fact that our 

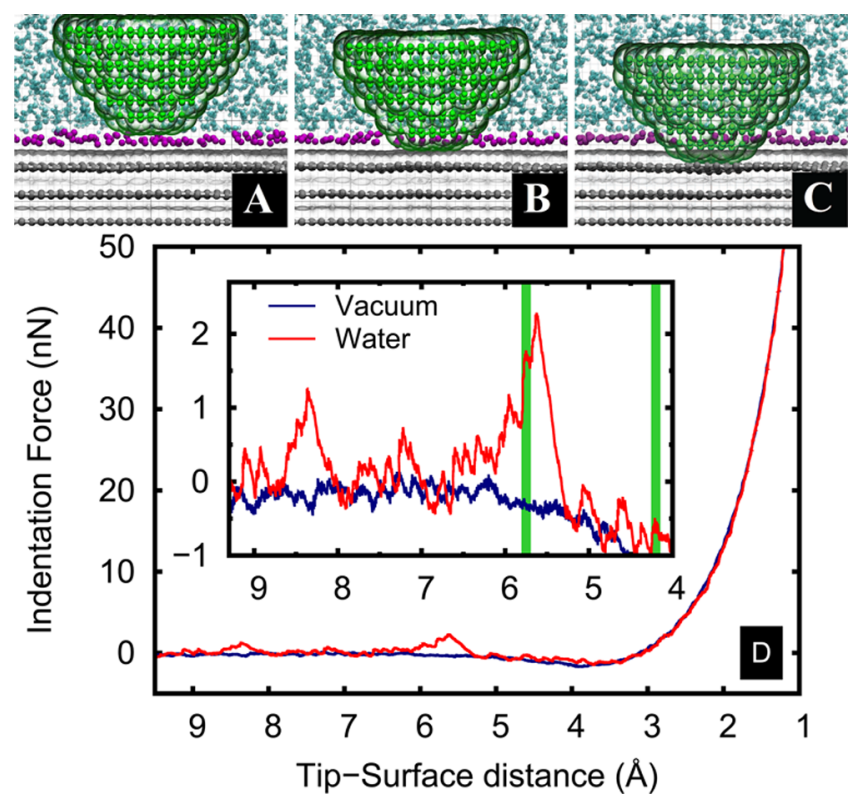

Figure 3. Simulations of indentation in water. Ball-and-stick models of the atomic configurations at different normal loads: (A) over the first hydration layer, (B) just after breaking it, (C) $F_{\mathrm{N}}=5 \mathrm{nN}$ (C). Gray, green, cyan, and purple represent graphene, diamond-tip, water, and first hydration layer water oxygen atoms, respectively. Besides the ball-and-stick models, we have also added the van der Waals surface $\left(r=\sigma_{\mathrm{cc}} / 2=1.65 \AA\right)$ of the tip (graphene substrate) represented by a transparent surface in green (gray). (D) Force felt by the tip as a function of the distance between its bottommost atom and the top layer of graphene. The inset highlights the force peaks associated with the breaking of the hydration layers. The green lines correspond to structures (A) and (B).

diamond tip is hydrophobic, ${ }^{29}$ and it is too small to form a structured (purely entropy driven) hydration layer. This possibly explains why we do not observe an attractive force regime right before each of the force peaks associated with the breaking of the hydration layers in the indentation force curve (see Figure 3D). This oscillatory behavior has been identified with hydrophilic tips ${ }^{30}$ and explained as a result of the interpenetration of the tip and surface hydration layers.

Figure 3D confirms that these layers are broken at rather low normal loads, in agreement with previous results obtained with dedicated frequency-modulation AFMs ${ }^{26}$ but extremely difficult to verify with standard commercial setups. Hence, it follows that in the range of $F_{\mathrm{N}}$ values where high-resolution images in water-FFM are achievable, the water layers are already broken, and therefore we are imaging with the tip in direct mechanical contact with the surface. Although this conclusion is somehow expected, taking into consideration the normal loads used in our experiments and the forces required to break the hydration layers formed over graphene, ${ }^{26}$ our MD simulations allow us to conclusively demonstrate that in our experiments we are imaging in direct mechanical contact.

The possible effect of the fluctuations induced by the fast collisions with the water molecules requires a fluid dynamics analysis. Recent MD simulations of pure water have shown, by integration of the autocorrelation stress tensor, that the viscosity as a function of time saturates in less than 1 ps. $^{31}$ Considering that the largest movement of the tip in $1 \mathrm{ps}$, i.e., during the slip phase, is $0.0012 \AA$, this would imply that in the time that one water molecule loses its stress memory the tip barely moves. We have explicitly confirmed this memory loss in our simulations, showing that after the slip phase only $5 \%$ of the water molecules initially in contact with the tip remained within the interacting distance (see Figure S5 in the Supporting Information). Thus, the stress imposed on the tip by the water results from purely random collisions with a null stress sum, i.e., fluctuations around zero. These random stress fluctuations can only be appreciated in a slight increase in the fluctuations of the friction force measured in water when compared with friction measured in vacuum at the same normal load (see Figure 2C).

Finally, we explore the information about the water structure and dynamics that could be obtained with AFM in the low-load regime, before breaking the hydration layer. To this end, we have simulated the friction force measured when the tip scans over this layer. The calculated force signal (Figure 2B) seems to resemble mostly white noise. However, computing its average (gray) curve in Figure 2B, we surprisingly recover a friction force that, although much lower $(\sim 0.1 \mathrm{nN})$ than the force curves obtained at high loads $(>5 \mathrm{nN})$, seems to display the same periodicity as graphene's hollow sites. This contrast appears inverted with respect to the high-load curves; that is, the maximum friction is obtained when the tip bottommost atoms are aligned with graphene's hollow sites. This result, at first surprising, can be understood in terms of recently published $a b$ initio results concerning the hydration properties of graphene. ${ }^{28}$ Although the first hydration layer is highly mobile, i.e., the water molecules are not anchored to any particular site in graphene, the probability of finding a water molecule on a hollow site is much higher than at any other site. $^{28}$ Therefore, when imaging the first hydration layer, i.e., a tip-water repulsive regime, one also expects that the friction will be higher at the sites where the water molecules are more likely to be found. This observation fully validates the hypothesis that the measurements in Figure 1 were performed with direct mechanical contact.

\section{CONCLUSIONS}

In summary, we have shown that friction measurements on graphene in water exhibit a high-resolution contrast almost identical to the one found in UHV. Extending the protocols for the simulation of friction, we report atomically detailed $\mathrm{MD}$ simulations of FFM in liquids. Our results link the experimental high resolution with the direct mechanical contact of tip and sample for loads larger than $5 \mathrm{nN}$ and demonstrate that water plays a purely stochastic role in this kind of measurement. In the low-load regime, our simulations provide a detailed characterization of the hydration layers formed in the graphene/water interface and its breaking process during indentation. Furthermore, they suggest that friction measurements could unveil the subtle dynamical lateral order induced by a hydrophobic material such as graphene on the water hydration layer. Among possible future applications, it would be interesting to extend this analysis to other systems where water intercalates and leads to considerable changes at the interface between graphene and the substrate. A representative process of this kind is the formation of oriented water stripes at the interface between graphene and mica. ${ }^{32}$ In this case, MD simulations could assess the stability of these systems when an external mechanical stress is applied to them. Our results together with recent findings ${ }^{33}$ on wet friction between sliding surfaces pave the way to nanoscale investigations of sliding friction in liquid environments other than water, with a broad range of possible applications, including the onset of corrosion and other tribochemical surface effects. Moreover, they 
promote water as an efficient alternative to UHV for reliable lattice resolution imaging and characterization of crystal surfaces and molecular coatings.

\section{METHODS}

Growth of Graphene on Copper (G/Cu) Sample. A $25 \mu \mathrm{m}$ thick, $5 \times 5 \mathrm{~cm}^{2}$ copper foil ( $99.8 \%$ purity, $25 \mu \mathrm{m}$ thick, Alfa Aesar) was polished using the electrochemical method, washed for $10 \mathrm{~min}$ by ultrapure water $(18 \mathrm{M} \Omega \cdot \mathrm{cm})$, and dried by a high-purity nitrogen flow for $5 \mathrm{~min}$. Then, the pretreated copper foil cut into $2 \times 2 \mathrm{~cm}^{2}$ was loaded into our homemade low-pressure chemical vapor deposition system in a multizone furnace (Lindberg Blue M HTF55667C), and the chamber was evacuated to $0.1 \mathrm{~Pa}$. The copper foil was annealed at $1020{ }^{\circ} \mathrm{C}$ by introducing hydrogen $(5 \mathrm{sccm}$, partial pressure of $\sim 10 \mathrm{~Pa})$ to remove the oxide of the surface. After that, methane $(10 \mathrm{ccm})$ was introduced into the chamber for $30 \mathrm{~min}$ to grow the intrinsic graphene film. Quenching the quartz tube, we quickly cooled it to $700{ }^{\circ} \mathrm{C}$. The growth was finished with hydrogen and cooling to room temperature in $10 \mathrm{~min}$.

Growth of Graphene on Nickel (G/Ni) Sample. The segregation growth method was used to obtain the graphene on a $\mathrm{Ni}$ substrate. First, $200 \mathrm{~nm}$ Ni films were deposited onto a $\mathrm{SiO}_{2} / \mathrm{Si}$ by e-beam evaporation system. Then, prepared $\mathrm{Ni}$ films were placed in vacuum annealing furnace (VTHK-350, Beijing Technol Science Co., Ltd.) with a chamber of $\Phi 120 \AA-\mathrm{L} 300 \mathrm{~mm}$ for graphene growth. The segregation process was as follows: (1) heating the substrate at $900-1100{ }^{\circ} \mathrm{C}$ after the chamber vacuum reaches $(0.4-4) \times 10^{-3} \mathrm{~Pa}$; (2) keeping the substrate for $100 \mathrm{~min}$ at the annealing temperature; (3) cooling to room temperature.

AFM Imaging. The FFM measurements were performed in two liquid cells filled with deionized water (Milli-Q Millipore with specific resistivity $18 \mathrm{M} \Omega \mathrm{cm}$ ) at room temperature. Two commercial AFM systems (Bruker Multimode III and Nanotec Cervantes) were used, with silicon cantilevers holding integrated ultrasharp tips (Bruker SNL$10 \mathrm{D}$ ). These sensors have nominal resonance frequencies of $18 \mathrm{kHz}$, a spring constant in the range $0.03-0.12 \mathrm{~N} / \mathrm{m}$, and a nominal tip radius of $2 \mathrm{~nm}$. The normal and lateral forces were calibrated using the method of Lüthi et al. ${ }^{34}$ The AFM images were processed using the WSxM software. ${ }^{35}$

Atomic Level Models and Force Fields. We have modeled our FFM measurements using a system composed of three parts: (1) the surface, a $5 \times 5 \mathrm{~nm}^{2}$ trilayer graphene slab with $A B A$ stacking; (2) the tip, a conically shaped diamond tip with a radius of $1.1 \mathrm{~nm}$ and a contact radius of $0.45 \mathrm{~nm}$ [the diamond is cleaved such that the (111) plane is parallel to the surface]; (3) the solvent; we solvate the system by adding 9300 water molecules. The atomic interactions are described by classical force fields $\left(\mathrm{GAFF}^{21}\right.$ for diamond and graphene and $\mathrm{TIP}_{4} \mathrm{P}^{36}$ for $\mathrm{H}_{2} \mathrm{O}$ ) that are known to accurately describe the mechanical ${ }^{27,37}$ and wetting ${ }^{36}$ properties of these materials.

MD Protocols. MD simulations were carried out using the AMBER14 program with NVIDIA-GPU acceleration. ${ }^{20,21}$ Simulations were performed using periodic boundary conditions and an integration time step of $1 \mathrm{fs}$. The particle-mesh-Ewald method was used to calculate long-range electrostatics and the van der Waals interactions with a cutoff of $10 \AA$. A constant temperature of $300 \mathrm{~K}$ was ensured in all the simulations by means of a Langevin thermostat. For the MD simulations performed in the NTP ensemble, a Berendsen barostat was used to keep the pressure constant at $1 \mathrm{~atm}$.

Our simulation protocol is composed of three main stages: (1) First we thermalize the system (equilibrate $T=300 \mathrm{~K}$ and $P=1 \mathrm{~atm}$ ) during $11 \mathrm{~ns}$ while restraining the tip-surface distance to $1 \mathrm{~nm}$; (2) then we indent, perpendicularly to the surface, in an NVT ensemble using constant velocity $(0.1 \mathrm{~m} / \mathrm{s})$ steered $\mathrm{MD}$; last we perform constant-velocity SMD $(0.1 \mathrm{~m} / \mathrm{s})$ to measure the friction force at different loads. For further details on indentation and lateral displacement SMD protocols refer to Figure S2 in the Supporting Information.

Evaluating Friction Forces from SMD Simulations. All the MD force curves shown in this work are time averages (over $25 \mathrm{ps)} \mathrm{of} \mathrm{the}$ instantaneous forces obtained from single SMD runs. We have confirmed (see Supporting Information) that this procedure, commonly used to measure friction in vacuum, ${ }^{19,22-24,33}$ is also able to account for the entropic contribution of friction in liquid environments. In fact, the maximum force deviation obtained from a single SMD curve and the force calculated using multiple SMD runs and the Jarzysnki equation estimated is $0.0175 \mathrm{nN}$ (see Supporting Information). The validity of this approach, i.e., approximating the free energy evolution by a single SMD work curve, stems from the slow speed of the SMD, which ensures that each run is a quasi-static process. Taking into account that this approximation is also known to correctly describe free energy evolution in much more complex biological systems, ${ }^{38}$ it comes as no surprise that it is also suitable to correctly describe friction processes.

\section{ASSOCIATED CONTENT}

\section{Supporting Information}

The Supporting Information is available free of charge on the ACS Publications website at DOI: 10.1021/acsnano.5b07825.

Further details concerning friction experiments at different loads, details of the MD simulation protocols, breaking of the hydration layer, and the rapid decay of the water stress memory (PDF)

\section{AUTHOR INFORMATION}

\section{Corresponding Author}

*E-mail: ruben.perez@uam.es.

\section{Notes}

The authors declare no competing financial interest.

\section{ACKNOWLEDGMENTS}

We acknowledge financial support from the Spanish MINECO (projects CSD2010-00024, MAT2011-023627, MAT20122448, FIS2012-36113-C03-03, MAT2013-44858-R, and MAT2014-54484-P), Comunidad de Madrid (project S2009/ MAT-1467), and MECD (Carlos Pimentel FPU grant). Computer time has been provided by the Red Española de Supercomputación (RES) at the Minotauro Supercomputer (BSC, Barcelona) and by the Extremadura Research Centre for Advanced Technologies (CETA-CIEMAT). Some AFM images and friction measurements were obtained at the ICTS Centro Nacional de Microscopia Electrónica (UCM). We gratefully thank Dr. Yu Zhou and Dr. Li Lin from the Beijing National Laboratory for Molecular Sciences (BNLMS) and the College of Chemistry and Molecular Engineering at Peking University for the graphene samples, and Rafael Buscalioni for valuable discussions. The COST Action MP1303 is gratefully acknowledged.

\section{REFERENCES}

(1) Binnig, G.; Quate, C. F.; Gerber, C. Atomic Force Microscope. Phys. Rev. Lett. 1986, 56, 930-933.

(2) Mate, C. M.; McClelland, G. M.; Erlandsson, R.; Chiang, S. Atomic-Scale Friction of a Tungsten Tip on a Graphite Surface. Phys. Rev. Lett. 1987, 59, 1942-1945.

(3) Meyer, E.; Gyalog, T.; Overney, R. M.; Dransfeld, K. Nanoscience: Friction and Rheology on The Nanometer Scale; World Scientific, 1998.

(4) Filleter, T.; Bennewitz, R. Structural and Frictional Properties of Graphene Films on $\mathrm{SiC}(0001)$ Studied by Atomic Force Microscopy. Phys. Rev. B: Condens. Matter Mater. Phys. 2010, 81, 155412.

(5) Maier, S.; Gnecco, E.; Baratoff, a.; Bennewitz, R.; Meyer, E. Atomic-Scale Friction Modulated by a Buried Interface: Combined Atomic and Friction Force Microscopy Experiments. Phys. Rev. B: Condens. Matter Mater. Phys. 2008, 78, 045432. 
(6) Goryl, M.; Budzioch, J.; Krok, F.; Wojtaszek, M.; Kolmer, M.; Walczak, L.; Konior, J.; Gnecco, E.; Szymonski, M. Probing AtomicScale Friction on Reconstructed Surfaces of Single-Crystal Semiconductors. Phys. Rev. B: Condens. Matter Mater. Phys. 2012, 85, 085308 .

(7) Steiner, P.; Gnecco, E.; Krok, F.; Budzioch, J.; Walczak, L.; Konior, J.; Szymonski, M.; Meyer, E. Atomic-Scale Friction on Stepped Surfaces of Ionic Crystals. Phys. Rev. Lett. 2011, 106, 186104.

(8) Ohnesorge, F.; Binnig, G. True Atomic Resolution by Atomic Force Microscopy Through Repulsive and Attractive Forces. Science 1993, 260, 1451-1456.

(9) Labuda, A.; Paul, W.; Pietrobon, B.; Lennox, R. B.; Grütter, P. H.; Bennewitz, R. High-Resolution Friction Force Microscopy under Electrochemical Control. Rev. Sci. Instrum. 2010, 81, 083701.

(10) Pina, C. M.; Miranda, R.; Gnecco, E. Anisotropic Surface Coupling While Sliding on Dolomite and Calcite Crystals. Phys. Rev. B: Condens. Matter Mater. Phys. 2012, 85, 073402.

(11) Nita, P.; Pimentel, C.; Luo, F.; Milián-Medina, B.; Gierschner, J.; Pina, C. M.; Gnecco, E. Molecular Resolution Friction Microscopy of $\mathrm{Cu}$ Phthalocyanine Thin Films on Dolomite (104) in Water. Nanoscale 2014, 6, 8334-8339.

(12) Novoselov, K. S.; Fal'ko, V. I.; Colombo, L.; Gellert, P. R.; Schwab, M. G.; Kim, K. A Roadmap for Graphene. Nature 2012, 490, 192-200.

(13) Robinson, B. J.; Kay, N. D.; Kolosov, O. V. Nanoscale Interfacial Interactions of Graphene with Polar and Nonpolar Liquids. Langmuir 2013, 29, 7735-7742.

(14) Egberts, P.; Ye, Z.; Liu, X. Z.; Dong, Y.; Martini, A.; Carpick, R. W. Environmental Dependence of Atomic-Scale Friction at Graphite Surface Steps. Phys. Rev. B: Condens. Matter Mater. Phys. 2013, 88, 035409.

(15) Egberts, P.; Han, G. H.; Liu, X. Z.; Johnson, A. T. C.; Carpick, R. W. Frictional Behavior of Atomically Thin Sheets: HexagonalShaped Graphene Islands Grown on Copper by Chemical Vapor Deposition. ACS Nano 2014, 8, 5010-5021.

(16) Paolicelli, G.; Tripathi, M.; Corradini, V.; Candini, A.; Valeri, S. Nanoscale Frictional Behavior of Graphene on $\mathrm{SiO} 2$ and $\mathrm{Ni}(111)$ Substrates. Nanotechnology 2015, 26, 055703.

(17) Lee, C.; Li, Q.; Kalb, W.; Liu, X.-Z.; Berger, H.; Carpick, R. W.; Hone, J. Frictional Characteristics of Atomically Thin Sheets. Science 2010, 328, 76-80.

(18) Deng, Z.; Smolyanitsky, A.; Li, Q.; Feng, X.-Q.; Cannara, R. J. Adhesion-Dependent Negative Friction Coefficient on Chemically Modified Graphite at The Nanoscale. Nat. Mater. 2012, 11, 10321037.

(19) Li, Q.; Liu, X.-Z.; Kim, S.-P.; Shenoy, V. B.; Sheehan, P. E.; Robinson, J. T.; Carpick, R. W. Fluorination of Graphene Enhances Friction Due to Increased Corrugation. Nano Lett. 2014, 14, 52125217.

(20) Götz, A. W.; Williamson, M. J.; Xu, D.; Poole, D.; Le Grand, S.; Walker, R. C. Routine Microsecond Molecular Dynamics Simulations with AMBER on GPUs. 1. Generalized Born. J. Chem. Theory Comput. 2012, 8, 1542-1555.

(21) Wang, J. M.; Wolf, R. M.; Caldwell, J. W.; Kollman, P. A.; Case, D. A. Development and Testing of a General Amber Force Field. J. Comput. Chem. 2004, 25, 1157-1174.

(22) Dong, Y.; Li, Q.; Martini, A. Molecular Dynamics Simulation of Atomic Friction: A Review and Guide. J. Vac. Sci. Technol., A 2013, 31, 030801.

(23) Dong, Y.; Wu, X.; Martini, A. Atomic Roughness Enhanced Friction on Hydrogenated Graphene. Nanotechnology 2013, 24, 375701.

(24) Li, Q.; Dong, Y.; Perez, D.; Martini, A.; Carpick, R. W. Speed Dependence of Atomic Stick-Slip Friction in Optimally Matched Experiments and Molecular Dynamics Simulations. Phys. Rev. Lett. 2011, 106, 126101.

(25) Dienwiebel, M.; Verhoeven, G. S.; Pradeep, N.; Frenken, J. W. M.; Heimberg, J. A.; Zandbergen, H. W. Superlubricity of Graphite. Phys. Rev. Lett. 2004, 92, 126101.
(26) Suzuki, K.; Oyabu, N.; Kobayashi, K.; Matsushige, K.; Yamada, $\mathrm{H}$. Atomic-Resolution Imaging of Graphite-Water Interface by Frequency Modulation Atomic Force Microscopy. Appl. Phys. Express 2011, 4, 125102.

(27) Zhou, H.; Ganesh, P.; Presser, V.; Wander, M. C. F.; Fenter, P.; Kent, P. R. C.; Jiang, D. E.; Chialvo, A. a.; McDonough, J.; Shuford, K. L.; Gogotsi, Y. Understanding Controls on Interfacial Wetting at Epitaxial Graphene: Experiment and Theory. Phys. Rev. B: Condens. Matter Mater. Phys. 2012, 85, 035406.

(28) Tocci, G.; Joly, L.; Michaelides, A. Friction of Water on Graphene and Hexagonal BN from Ab-Initio Methods: Very Different Slippage Despite Very Similar Interface Structures. Nano Lett. 2014, $14,6872-6877$.

(29) Horinek, D.; Serr, A.; Geisler, M.; Pirzer, T.; Slotta, U.; Lud, S. Q.; Garrido, J. A.; Scheibel, T.; Hugel, T.; Netz, R. R. Peptide Adsorption on a Hydrophobic Surface Results from an Interplay of Solvation, Surface, and Intrapeptide Forces. Proc. Natl. Acad. Sci. U. S. A. 2008, 105, 2842-2847.

(30) Fukuma, T.; Reischl, B.; Kobayashi, N.; Spijker, P.; Canova, F. F.; Miyazawa, K.; Foster, A. S. Mechanism of Atomic Force Microscopy Imaging of Three-Dimensional Hydration Structures at a Solid-Liquid Interface. Phys. Rev. B: Condens. Matter Mater. Phys. 2015, 92, 155412.

(31) Tazi, S.; Botan, A.; Salanne, M.; Marry, V.; Turq, P.; Rotenberg, B. Diffusion Coefficient and Shear Viscosity of Rigid Water Models. J. Phys.: Condens. Matter 2012, 24, 284117.

(32) Kim, J.-S.; Choi, J. S.; Lee, M. J.; Park, B. H.; Bukhvalov, D.; Son, Y.-W.; Yoon, D.; Cheong, H.; Yun, J.-N.; Jung, Y.; Park, J. Y.; Salmeron, M. Between Scylla and Charybdis: Hydrophobic GrapheneGuided Water Diffusion on Hydrophilic Substrates. Sci. Rep. 2013, 3, 2309.

(33) Chen, W.; Foster, A. S.; Alava, M. J.; Laurson, L. Stick-Slip Control in Nanoscale Boundary Lubrication by Surface Wettability. Phys. Rev. Lett. 2015, 114, 095502.

(34) Lüthi, R.; Meyer, E.; Haefke, H.; Howald, L.; Gutmannsbauer, W.; Guggisberg, M.; Bammerlin, M.; Güntherodt, H.-J. Nanotribology: an UHV-SFM Study on Thin Films of C60 and AgBr. Surf. Sci. 1995, $338,247-260$.

(35) Horcas, I.; Fernández, R.; Gómez-Rodríguez, J. M.; Colchero, J.; Gómez-Herrero, J.; Baro, a. M. WSXM: A Software For Scanning Probe Microscopy and a Tool for Nanotechnology. Rev. Sci. Instrum. 2007, 78, 013705.

(36) Abascal, J. L.; Vega, C. A General Purpose Model for The Condensed Phases of Water: TIP4P/2005. J. Chem. Phys. 2005, 123, 234505.

(37) Tsai, J.-L.; Tu, J.-F. Characterizing Mechanical Properties of Graphite Using Molecular Dynamics Simulation. Mater. Eng. 2010, 31, 194-199.

(38) Park, S.; Khalili-Araghi, F.; Tajkhorshid, E.; Schulten, K. Free Energy Calculation from Steered Molecular Dynamics Simulations Using Jarzynskis Equality. J. Chem. Phys. 2003, 119, 3559-3566. 